\title{
Eficiencia de trampas de caída y suficiencia taxonómica en comunidades de arañas (Araneae) epigeas en tres ecorregiones del noroeste argentino
}

\author{
Ivanna Gabriela Cruz ${ }^{1}$, Victor Manuel Torres ${ }^{1}$, Andrea Ximena González-Reyes ${ }^{2}$ \\ \& José Antonio Corronca ${ }^{1}$ \\ 1. Consejo Nacional de Investigaciones Científicas y Técnicas (CONICET), Instituto para el Estudio de la Biodiversidad \\ de Invertebrados (IEBI), Universidad Nacional de Salta (UNSa). Av. Bolivia 5150. Salta, Argentina; \\ ivannagabrielacruz@gmail.com, torres.vicman@gmail.com, jcorronca@gmail.com \\ 2. IEBI-UNSa. Av. Bolivia 5150. Salta, Argentina; andyximena@gmail.com
}

Recibido 02-V-2017. Corregido 10-X-2017. Aceptado 09-XI-2017.

\begin{abstract}
Efficiency of pitfall traps and taxonomic sufficiency for epigeal spider communities (Araneae) in three ecoregions of northwestern Argentina. The increased degradation of natural habitats has strengthened the need to know and assess biodiversity patterns. Particularly, the study of the spider fauna in the North of Argentina is scarce in ecoregions with priority interests of conservation. Generally, spiders are used as indicators to compare biodiversity patterns, and here we tested whether the spider family-level can act as a substitute of the species-level in biodiversity rapid assessments. For this, we analyzed the alpha and beta diversity of the epigeal spider communities in three separate sites of three ecoregions of Salta province (Chaco Serrano, Monte de Sierras and Bolsones, and Puna), during the fall, winter, spring and summer of 2005-2007. In each site, 10 pitfall traps, located along a linear transect and 10 meters apart, were placed for seven days per season of continuous activity. A total of 886 spiders were collected from 100 species/morphospecies of 19 families. The completeness of the inventory obtained for each ecoregion surpassed $70 \%$. The Chaco ecoregion $(\mathrm{S}=56, \mathrm{~N}=495)$ reported the highest species richness and abundance compared to Monte $(\mathrm{S}=44, \mathrm{~N}=262)$ and Puna $(\mathrm{S}=23, \mathrm{~N}=129)$. Alpha and beta diversity showed that ecoregional spider communities were different, sharing few species $(0.7$ $\%)$. The Chaco reported a high dissimilarity of its assemblage with respect to the other ecoregions. The colder seasons (autumn and winter) are important in assessing the diversity of spiders, contributing to regional diversity in conjunction with the diversity of warm seasons (spring and summer). Four guilds were reported (ground hunters, specialists, other hunters and ambush hunters), but the latter was absent in Monte, and the specialists dominated Chaco. Zodariidae was dominant in Chaco Serrano, where Leprolochus birabeni is an indicator of native environments. On the other hand, Lycosidae, Philodromidae, Anyphaenidae and Oonopidae were important for Monte and Puna. Year-round pitfall traps and identification of epigeal spider families are useful for biodiversity rapid assessments in this area. Like the species-level, the taxonomic family-level evidenced changes in alpha and beta diversity. This allows the inclusion of this taxonomic group for future biodiversity monitoring.

Rev. Biol. Trop. 66(1): 204-217. Epub 2018 March 01.
\end{abstract}

Key words: spider's diversity, pitfall traps, ecoregions, seasonality, taxonomic sufficiency.

El incremento de la degradación y la destrucción de los hábitats naturales ha fortalecido la necesidad de conocer y evaluar los patrones de biodiversidad y su variación espacio-temporal con el propósito de su conservación y manejo (Hsieh, Lin, \& Tso, 2003). En este sentido, las ecorregiones son cada vez más aceptadas como unidades geográficas útiles para la planificación de estrategias de conservación a escala regional y global (Magnusson, 2004). Ellas son definidas como unidades relativamente grandes de tierra o agua que contienen un conjunto característico de comunidades naturales que comparten una gran mayoría de especies, dinámicas y condiciones ambientales (Olson \& Dinerstein, 1998). En Argentina, las ecorregiones Puna, Monte de Sierras y Bolsones y Chaco Serrano son consideradas zonas 
prioritarias para su conservación. La Puna, una altiplanicie extensa que ocupa parte de Argentina, Bolivia, Chile y Perú (Cabrera, 1957), ha sido incluida en el Global 200 (estado de conservación vulnerable) debido al alto valor endémico de su flora y fauna (Olson \& Dinerstein, 2002); al igual que la ecorregión Monte de Sierras y Bolsones. Esta última no se comparte con ningún otro país, por lo que la Argentina es la única responsable de su conservación (Bertonatti \& Corcuera, 2000). Por su lado, el Chaco Serrano que se extiende hasta pequeños sectores en el sur de Bolivia se encuentra entre los biomas más amenazados del mundo (Grau, Gasparri, \& Aide, 2008), y ocupa el primer lugar de las ecorregiones para conservar en la Argentina debido a la fuerte reducción y fragmentación que sufren sus hábitats (PiquerRodríguez et al., 2015).

Los inventarios biológicos constituyen una manera de describir y monitorear la diversidad (Whitmore, Crouch, \& Slotow, 2002) y permiten evaluar la riqueza de especies en un área determinada (Cardoso, Silva, De Oliveira, \& Serrano, 2007). Debido a que los estudios exhaustivos y detallados de biodiversidad requieren una elevada inversión económica y de tiempo, es necesario planificar protocolos que reduzcan el tiempo y el esfuerzo de muestreo, permitiendo a su vez realizar evaluaciones rápidas de diversidad y un monitoreo de sus especies (Porto, Pequeño, \& Tourinho, 2016). Por otro lado, la estacionalidad es un factor importante que puede afectar tanto la estructura y la composición de las comunidades como el estudio de los patrones de diversidad (Moreno \& Halffter, 2000). Por lo cual, los planes de muestreo también deben cubrir todas las estaciones del año para lograr una buena estimación de la composición de los ensambles de especies y analizar su dinámica temporal (Hsieh \& Linsenmair, 2011).

Otra herramienta eficaz en el contexto de las evaluaciones rápidas de biodiversidad es la suficiencia taxonómica, la cual se define como la identificación de los organismos a una resolución taxonómica suficiente para satisfacer los objetivos de un estudio (Ellis, 1985). Así, el análisis de las comunidades a nivel de género, subfamilias, familias e incluso phylum, puede ser útil en estudios rápidos de biodiversidad. Muchos autores han demostrado respuestas satisfactorias del empleo de esta herramienta para monitoreos en cortos períodos de tiempo y estrategias de conservación en un área pobremente conocida y con recursos monetarios y logísticos limitados (Pik, Oliver, \& Beattie, 1999; Brennan, Ashby, Majer, Moir \& Koch, 2006; Groc et al., 2010; Timms, Bowden, Summerville, \& Buddle, 2013; Muñoz-Gutiérrez, Roussea, Andrade-Silva, \& Delabie, 2017). Este enfoque contribuye a superar el impedimento taxonómico en varios grupos de invertebrados terrestres, tales como las arañas (Brennan et al., 2006; Landeiro et al., 2012). Ellas constituyen un grupo de artrópodos depredador con una elevada diversidad de especies que produce un fuerte impacto en los ecosistemas terrestres (Grismado, Ramírez, \& Izquierdo, 2014), por lo que representan un taxón indicador potencialmente útil para comparar patrones de biodiversidad (Hsieh et al., 2003; Buchholz, 2010; Pedley et al., 2016). Sin embargo, son frecuentemente ignorados en los planes de manejo y monitoreo ambiental (New, 1996) debido a la dificultad para llegar a una determinación taxonómica completa. No obstante el nivel taxonómico de familia y el estudio de los gremios pueden potencialmente servir como sustitutos ecológicos para las especies (Cardoso, Pekár, Jocque, \& Coddington, 2011), ya que proveen una indicación de la composición en relación a la diversidad en el uso del hábitat y de estrategias de forrajeo (Haddad, Honiball, Dippenaar-Schoeman, Slotow, \& Van Rensburg, 2009; Cardoso et al., 2011).

El estudio de la araneofauna en las ecorregiones del norte Argentino es escaso, solo se conocen los estudios de González-Reyes, Corronca y Arroyo (2012) y Cava, Corronca \& Echeverría (2013) quienes destacan a las arañas como uno de los órdenes más abundantes de la comunidad de artrópodos en el Monte de Sierras y Bolsones, y la Puna, de la provincia de Salta. Por ello, el objetivo de este trabajo fue analizar la diversidad alfa y beta 
de las comunidades de arañas epigeas, en el Chaco Serrano, Monte de Sierras y Bolsones, y Puna, de la provincia de Salta, mediante un esquema estandarizado de muestreo considerando las cuatro estaciones del año. En segundo lugar, se evaluó si el nivel taxonómico de familia refleja patrones similares de diversidad alfa y beta en dichas ecorregiones, con el propósito de ser utilizadas como posible sustituto de la diversidad de especies en evaluaciones rápidas de biodiversidad.

\section{MATERIALES Y MÉTODOS}

Área de estudio: Se seleccionaron 3 sitios de muestreo para cada una de las ecorregiones de Chaco Serrano (Chaco), Monte de Sierras y Bolsones (Monte) y Puna de la provincia de Salta (Fig. 1). Los sitios de Chaco se caracterizan por presentar un clima subtropical cálido con temperatura media anual de $23{ }^{\circ} \mathrm{C}$ (Chébez, 2005) y promedios máximos y mínimos que oscilan entre 26 y $10{ }^{\circ} \mathrm{C}$, respectivamente

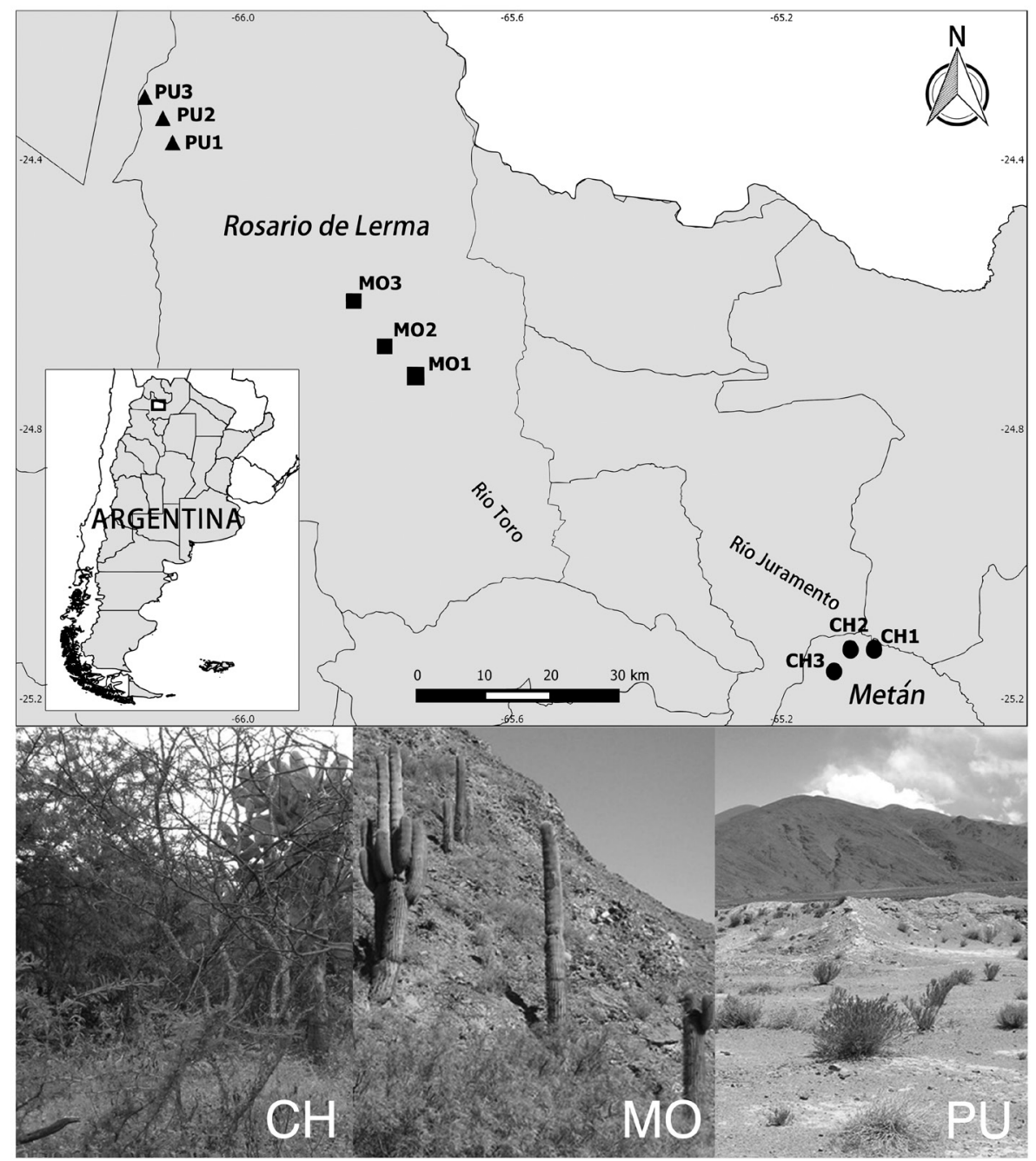

Fig. 1. Área de estudio y sitios de muestreo en las ecorregiones Chaco Serrano (CH), Monte de Sierras y Bolsones (MO), y Puna (PU), Salta, Argentina.

Fig. 1. Location of the study area and samples sites in Chaco Serrano $(\mathrm{CH})$, Monte de Sierras y Bolsones (MO) and Puna (PU) ecoregions of Salta, Argentina. 
(Moglia \& Giménez, 1998). La ecorregión presenta períodos secos (otoño e invierno) y lluviosos (primavera y verano), alcanzando valores de precipitación anual entre los 500$700 \mathrm{~mm}$ (Morello, 2012). La vegetación está representada por un bosque seco subtropical con una alta diversidad florística. Las especies dominantes son Schinopsis haenckeana Engl. (horco-quebracho), Aspidosperma quebrachoblanco Schlecht (quebracho blanco) y Prosopis alba Griseb y Prosopis nigra Griseb (algarrobos) (Morello, 2012).

El Monte de Sierras y Bolsones muestra una gran variedad de estructuras geomorfológicas y altitudes. El clima es semiárido a árido, con temperaturas media anual de 13-18 ${ }^{\circ} \mathrm{C}$ (Rundel, Villagra, Dillon, Roig-Juñent, \& Debandi, 2007) y marcados cambios tanto diarios como estacionales en la temperatura (Bertonatti \& Corcuera, 2000). Las lluvias son escasas con un promedio anual entre 100-350 mm (Labraga \& Villalba, 2009), concentradas en el verano. Los suelos son arenosos, pedregosos y salinos, pobres en materia orgánica, con frecuentes afloramientos rocosos. Larrea spp. (jarillal) caracterizan la unidad fitosociológica de esta ecorregión, formando matorrales de 1.5$3.0 \mathrm{~m}$ de altura (Bertonatti \& Corcuera, 2000).

Los sitios muestreados de la ecorregión Puna, corresponden a un desierto frío a $3000-4$ $000 \mathrm{~m}$ de altura. La sequedad y temperaturas extremas caracterizan a esta ecorregión, donde las temperaturas mínimas del invierno pueden llegar a los $-15^{\circ} \mathrm{C}$; con una gran amplitud térmica diaria, en algunos casos más de $25^{\circ} \mathrm{C}$ en el verano (Reboratti, 2006). Las escasas precipitaciones (principalmente estivales), alcanzan los $130 \mathrm{~mm}$ anuales (Mosca Torres \& Puig, 2010). Los suelos pobremente desarrollados (arenosos, pedregosos o salinos, muy susceptibles a la erosión), presentan un bajo porcentaje de materia orgánica y cobertura vegetal (Reboratti, 2006). Especies de Poa, Festuca y Stipa (pastos), Paraestrephia (tola), y Azorella yareta Hauman (yareta) (Bertonatti \& Corcuera, 2000) son abundantes en esta ecorregión.
Muestreo: Los sitios geo-referenciados en cada ecorregión estuvieron separados entre sí por al menos tres kilómetros (Fig. 1). Tres sitios del Chaco se ubicaron en el departamento Metán (CH1: 755 msnm (25 7’31.44" S \& 65 2'16.92" W), CH2: 813 msnm (25 7'22.8" $\mathrm{S} \& 65^{\circ} 3$ '44.4" W) y CH3: $829 \mathrm{msnm}\left(25^{\circ}\right.$ 7'25.2" S \& 65 5'52.8" W), mientras que los sitios pertenecientes al Monte y la Puna se localizaron en el departamento de Rosario de Lerma (MO1: 2417 msnm (24 41'34.08" $\left.\mathrm{S} \& 65^{\circ} 46^{\prime} 1.92^{\prime \prime} \mathrm{W}\right), \mathrm{MO} 2: 2467 \mathrm{msnm}\left(24^{\circ}\right.$ 40'36.84" S \& 65 47'21.12" W), MO3: 2554

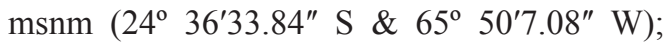
PU1: 3705 msnm $\left(24^{\circ} 22^{\prime} 27.84^{\prime \prime} \mathrm{S} \& 66^{\circ}\right.$

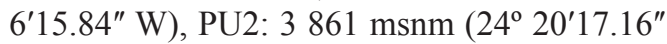
$\left.\mathrm{S} \& 66^{\circ} 7^{\prime} 8.04^{\prime \prime} \mathrm{W}\right)$ y PU3: $3989 \mathrm{msnm}\left(24^{\circ}\right.$ $\left.18^{\prime} 24.12^{\prime \prime} \mathrm{S} \& 66^{\circ} 8^{\prime} 44.16^{\prime \prime} \mathrm{W}\right)$. En cada uno de ellos se realizaron muestreos estacionales: en los meses de abril (otoño), agosto (invierno) y noviembre (primavera) de 2006, y marzo (verano) de 2007 para el Chaco; mientras que para el Monte y la Puna se muestreó durante los meses de noviembre (primavera) de 2005, febrero (verano), mayo (otoño) y agosto (invierno) de 2006. Se utilizó trampas de caída como método de recolección de arañas epígeas porque es muy usado en estudios faunísticos y ecológicos por ser fáciles de manipular y su bajo costo (Siewers et al., 2014), capturando un alto número de individuos y especies que permiten aplicar análisis estadísticos (Spence \& Niemelä, 1994). En cada sitio se colocaron al azar diez trampas de caída separadas entre sí por $10 \mathrm{~m}$ a lo largo de una transecta lineal, y fueron retiradas a los siete días de actividad por estación. Las trampas estuvieron representadas por recipientes plásticos de $7.5 \times 12.2 \times 5.2 \mathrm{~cm}$ (diámetro superior $\mathrm{x}$ profundidad $\mathrm{x}$ diámetro inferior), con solución salina (sal $(\mathrm{kg})$ : agua (l) en proporción 1:8, con gotas de detergente). El material recolectado fue preservado en etanol al $70 \%$ e identificado en el laboratorio de Artrópodos del IEBI-Facultad de Ciencias Naturales, Universidad Nacional de Salta entre los años 2007-2015. 
Identificación del material: Las arañas fueron identificadas a nivel de familia utilizando claves taxonómicas (Grismado et al., 2014) $\mathrm{y}$ posteriormente a nivel de género $\mathrm{y} / \mathrm{o}$ especie teniendo en cuenta las revisiones de géneros y las descripciones de especies, siguiendo la nomenclatura del World Spider Catalog (2017). Cuando no fue posible la identificación específica, las arañas fueron clasificadas en morfoespecies en base a caracteres estructurales diferenciables (Krell, 2004; Escorcia, Martínez, \& Silva, 2012; Rodriguez-Artigas, Ballester, \& Corronca, 2016). Para mayor simplicidad de aquí en adelante, el término especies será referido tanto para las morfoespecies como para los ejemplares identificados a nivel específico. Asimismo, a los ejemplares inmaduros que fueran recolectados en la misma trampa con los adultos identificados, se les asignó mediante comparaciones morfológicas (patrón de coloración, disposición y número de espinas en las patas, entre otros), ya que su inclusión fue necesaria para obtener estimaciones confiables de la riqueza de especies (Jiménez-Valverde \& Lobo, 2007; Rubio \& Moreno, 2010; Castanheira, Pérez-González, \& Baptista, 2016). Posteriormente, las arañas fueron clasificadas en gremios siguiendo a Cardoso et al. (2011). Los especímenes recolectados fueron depositados en la colección del Instituto para el Estudio de la Biodiversidad de Invertebrados - Museo de Ciencias Naturales, Universidad Nacional de Salta (IEBI-MCN- \#0003-000897-001082).

Diversidad alfa: Se calculó la riqueza de especies, abundancia de individuos, número de familias y número de especies representadas por uno y dos individuos (singletones y dobletones, respectivamente) por sitio, estación y ecorregión. La eficiencia del muestreo fue evaluada como el porcentaje de especies observadas con respecto a las esperadas por el estimador no paramétrico Chaol (Cardoso et al., 2008) mediante el programa EstimateS 9.1 (Colwell, 2013). Los cambios ecorregionales y estacionales de la riqueza de especies fueron evaluados mediante el análisis de rarefacciónextrapolación basado en individuos a un mismo nivel de cobertura de la muestra, con intervalos de confianza (IC) del $95 \%$ y 100 permutaciones utilizando el programa iNEXT (Chao, Ma, \& Hsieh, 2016). Este método asegura que las muestras sean comparadas con igual completitud, independientemente del tamaño de la muestra, lo cual permite realizar inferencias más robustas acerca de los patrones de riqueza de especies de las comunidades (Chao \& Jost, 2012).

Diversidad beta: Se determinó el porcentaje de fauna compartida entre las ecorregiones utilizando el índice de disimilitud de Morisita para tres $\left(\mathrm{C}_{33}\right)$ y dos $\left(\mathrm{C}_{22}\right)$ comunidades, calculado por medio del programa SPADE (Chao \& Shen, 2003-2005). Para evaluar si existieron diferencias de significación estadística entre los ensambles de especies por ecorregión y por estación, se utilizó el Procedimiento de Permutación de Respuesta Múltiple (MRPP) usando Bray-Curtis como medida de distancia, por medio del programa PC-Ord 5.0 (McCune \& Mefford, 1999). El mismo análisis fue aplicado para los ensambles de familias y gremios. Posteriormente, para evaluar la suficiencia taxonómica a nivel de familias, se correlacionaron los valores de riqueza de especies y de familias por medio del coeficiente de correlación de Pearson (diversidad alfa), y se realizó un Test de Mantel, para correlacionar las matrices de similitud (Bray-Curtis) de los ensambles de especies y familias (diversidad beta) (Pik et al., 1999; Groc et al., 2010). Ambos análisis fueron realizados por medio del programa PAST ver. 3.11 (Hammer, Harper, \& Ryan, 2001).

\section{RESULTADOS}

Diversidad alfa: Un total de 886 individuos de arañas epigeas fueron recolectadas pertenecientes a 100 especies, y 19 familias (Apéndice digital). Leprolochus birabeni (Zodariidae) (45\%), Lycosidae sp.1 (9\%) y Aillutticus pinquidor (Salticidae) (8\%) fueron especies dominantes para el Chaco; Agalenocosa sp. (Lycosidae) (23\%), Lycosidae sp.2 (14\%) y Cybaeodamus ornatus (Zodariidae) 
(8 \%) para el Monte; mientras que Petrichus sp.1 (Philodromidae) (19\%), Unicorn sikus (Oonopidae) (19\%) y Sanogasta maculatipes (Anyphaenidae) (14 \%) para la Puna. Los inventarios obtenidos para las tres ecorregiones lograron una completitud en el orden del 71 $\%$ para el Chaco y superiores al $80 \%$ para el Monte y la Puna (Cuadro 1). Por otro lado, la primavera y el verano evidenciaron los valores más altos de completitud del inventario para el Chaco y el Monte; mientras que para la Puna lo fue el verano seguido del invierno (Cuadro 2).

La ecorregión Chaco reportó el mayor número de especies e individuos seguido del Monte y la Puna (Cuadro 1). Sin embargo, al comparar la riqueza de especies a un mismo nivel de cobertura de muestreo, no se observaron diferencias de significación estadística entre el Chaco y el Monte ( $\mathrm{P} \geq 0.05$ ) (Fig. 2). Por otro lado, el verano seguido por el otoño, presentaron la mayor riqueza de especies en las tres ecorregiones (Cuadro 2). Al comparar estos valores a un mismo nivel de cobertura de muestreo, el otoño en el Chaco y Monte presentó un mayor número de especies. Mientras que para la Puna, lo fueron el otoño, la primavera y el verano (Fig. 2).

Diversidad beta: El $0.7 \%\left(\mathrm{C}_{33}=0.007\right)$ del total de las especies registradas fueron comunes a las tres ecorregiones. El Chaco reportó una alta disimilitud de su ensamble con respecto al Monte $\left(1-\mathrm{C}_{22}=0.996\right)$ y la Puna $\left(1-\mathrm{C}_{22}=0.996\right)$, compartiendo siete $\mathrm{y}$ dos especies respectivamente; mientras que 15 especies fueron compartidas entre el Monte y la Puna, con una similitud del orden del $14.6 \%$ $\left(1-\mathrm{C}_{22}=0.854\right) . \mathrm{El}$ análisis de procedimiento de permutación de respuesta múltiple (MRPP) corroboró que los ensambles de cada ecorregión fueron diferentes $\left(\mathrm{A}_{\text {Chaco-Monte }}=0.27, \mathrm{P}=\right.$ $0.02 ; \mathrm{A}_{\text {Chaco-Puna }}=0.32, \mathrm{P}=0.02 ; \mathrm{A}_{\text {Monte-Puna }}=$ $0.11, \mathrm{P}=0.02)$. Por su parte, la estacionalidad contribuyó al patrón de diversidad en las tres ecorregiones. Así, el otoño y el invierno del Chaco mostraron poseer una composición de especies diferente a la primavera y el verano (MRPP: $\mathrm{A}_{\text {Otoño-Primavera }}=0.16, \mathrm{P}=0.02$;

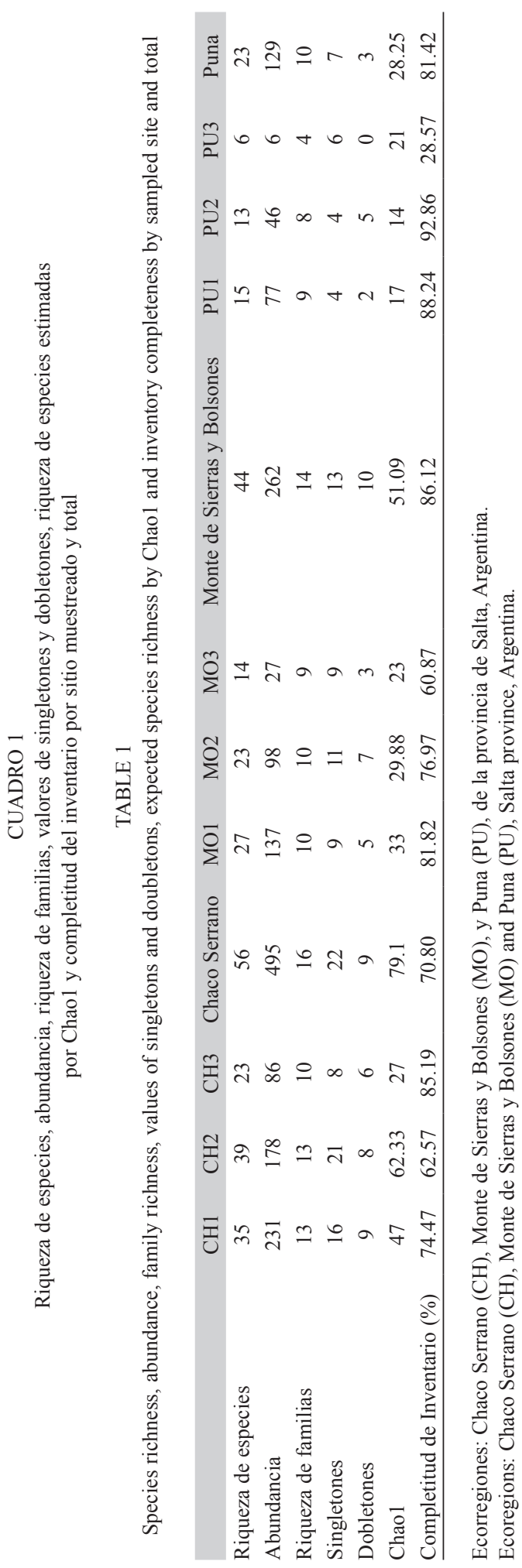




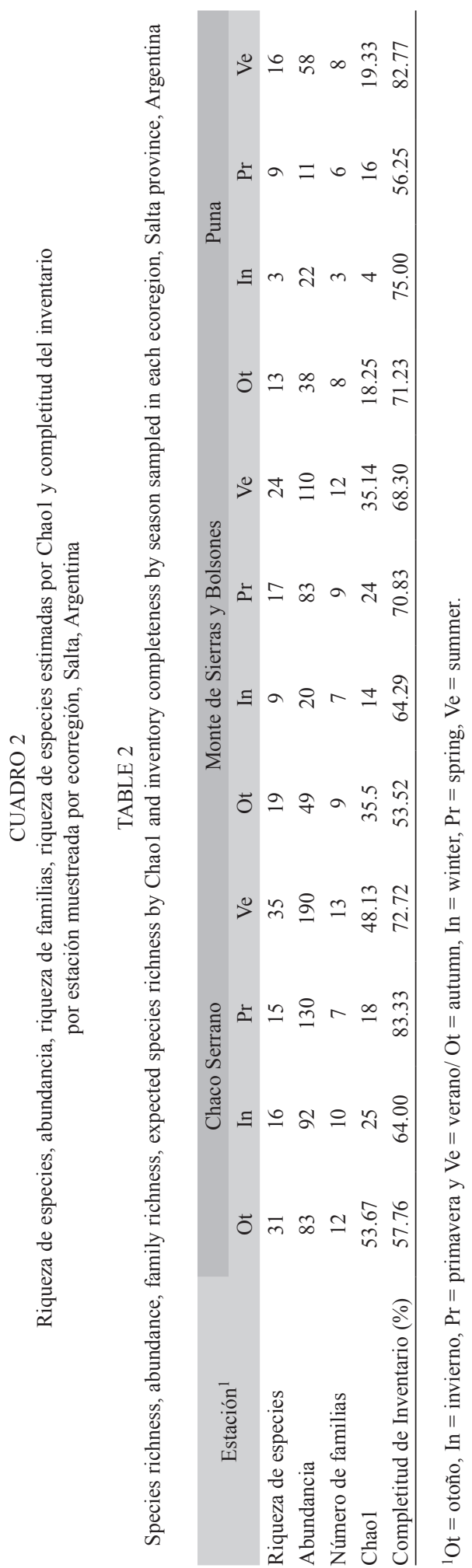

$\mathrm{A}_{\text {Invierno-Verano }}=0.13, \mathrm{P}=0.02$ ), donde también estas dos últimas mostraron diferencias de significación estadística (MRPP: $\mathrm{A}_{\text {Primavera-Verano }}=$ $0.09, \mathrm{P}=0.02$ ). El Monte y la Puna presentaron un patrón diferente al Chaco, donde el invierno exhibió un ensamble de especies particular con respecto a las demás estaciones $\left(\mathrm{MRPP}_{\text {Monte }}\right.$ : $\mathrm{A}_{\text {Invierno-Otoño }}=0.18, \mathrm{P}=0.05 ; \mathrm{A}_{\text {Invierno-Primavera }}$ $=0.17, \mathrm{P}=0.04 ; \mathrm{A}_{\text {Invierno-Verano }}=0.18, \mathrm{P}=$ $0.03 ; \mathrm{MRPP}_{\text {Puna }}: \mathrm{A}_{\text {Invierno-Otoño }}=0.21, \mathrm{P}=0.02$; $\mathrm{A}_{\text {Invierno-Primavera }}=0.12, \mathrm{P}=0.03 ; \mathrm{A}_{\text {Invierno-Verano }}$ $=0.26, \mathrm{P}=0.02$ ).

Composición de familias, gremios y suficiencia taxonómica: La composición de familias fue significativamente diferente entre las ecorregiones estudiadas $\left(\mathrm{A}_{\text {Chaco-Monte }}=0.16, \mathrm{P}\right.$ $<0.01 ; \mathrm{A}_{\text {Chaco-Puna }}=0.30, \mathrm{P}<0.01 ; \mathrm{A}_{\text {Monte-Puna }}$ $=0.12, \mathrm{P}=0.01)$. La suficiencia taxonómica de este nivel taxonómico fue congruente con el de especies en el patrón de diversidad alfa ( $\mathrm{r}$ $=0.93, \mathrm{P}<0.01)$ y beta (Test de Mantel: $\mathrm{R}=$ $0.70, \mathrm{P}<0.01)$. Cuatro gremios fueron registrados en el área de estudio: cazadoras de suelo, especialistas, otras cazadoras y cazadoras por emboscada, de los cuales este último estuvo ausente en el Monte (Fig. 3). El MRPP solo evidenció cambios significativos en el Chaco comparado con el Monte y la Puna $\left(\mathrm{A}_{\text {Chaco-Monte }}\right.$ $=0.48, \mathrm{P}=0.02 ; \mathrm{A}_{\text {Chaco-Puna }}=0.52 ; \mathrm{P}=0.02$; $\left.\mathrm{A}_{\text {Monte-Puna }}=0.02, \mathrm{P}=0.53\right)$. Así, las especialistas fueron dominantes en el Chaco, disminuyendo marcadamente su abundancia en el Monte, y en la Puna. Este patrón se invierte al considerar las otras cazadoras, que presentaron baja abundancia en el Chaco pero incrementaron su número en el Monte y en la Puna donde fueron dominantes. Por último, las cazadoras de suelo dominaron en el Monte, mostrando una menor abundancia en el Chaco (Fig. 3).

\section{DISCUSIÓN}

En este estudio se comparan los ensambles de arañas epigeas en tres ecorregiones con prioridad de conservación para la provincia de Salta y el Noroeste Argentino. La eficacia de las trampas de caída como método de muestreo 


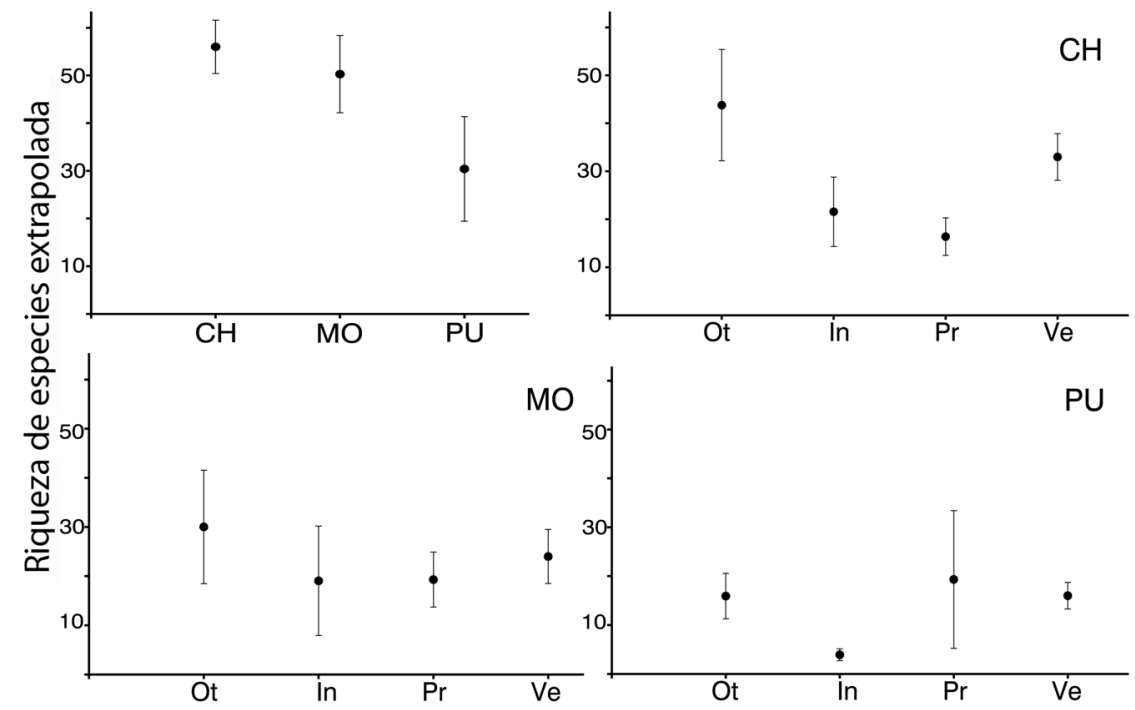

Fig. 2. Análisis de rarefacción-extrapolación basado en individuos por ecorregión: Chaco Serrano $(\mathrm{CH})$, Monte de Sierras y Bolsones (MO) y Puna (PU), Salta, Argentina. Riqueza de especies de arañas epigeas por ecorregión (a) y estación (b-d). Las barras indican los intervalos de confianza del $95 \%$. Ot = otoño, In = invierno, $\mathrm{Pr}=$ primavera, $\mathrm{Ve}=$ verano.

Fig. 2. Analysis of rarefaction-extrapolation based in individuals by ecorregion: Chaco Serrano (CH), Monte de Sierras and Bolsones (MO) and Puna (PU) of Salta, Argentina. The epigeal spider species richness by ecoregion (a) and seasons (b-d). The bars indicate the confidence intervals of $95 \%$. Ot $=$ autumn, $\mathrm{In}=$ winter, $\mathrm{Pr}=$ spring, $\mathrm{Ve}=$ summer.

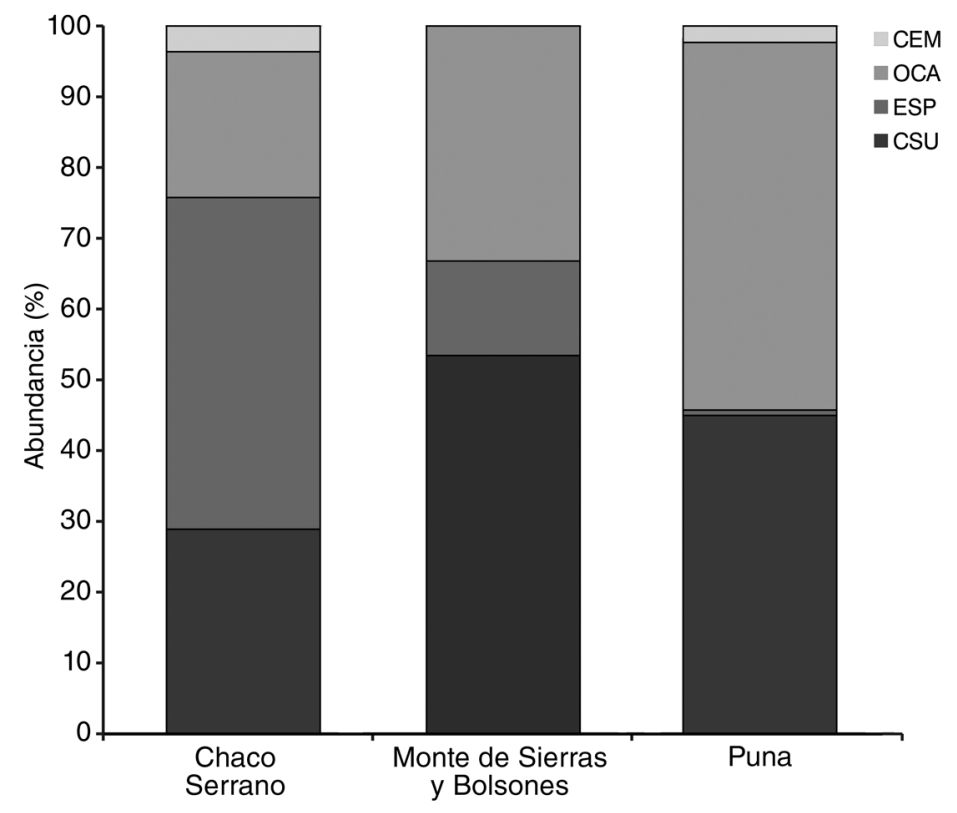

Fig. 3. Proporción de gremios de arañas epigeas en las ecorregiones: Chaco Serrano, Monte de Sierras y Bolsones, y Puna, Salta, Argentina. $\mathrm{CSU}=$ cazadoras de suelo, ESP = especialistas, OCA = otras cazadoras, $\mathrm{CEM}=$ cazadoras por emboscada. Fig. 3. Proportion of epigeal spider guilds in Chaco Serrano, Monte de Sierras y Bolsones and Puna ecoregions of Salta province, Argentina. $\mathrm{CSU}=$ ground hunters, $\mathrm{ESP}=$ specialists, $\mathrm{OCA}=$ other hunters, $\mathrm{CEM}=$ ambush hunters. 
se ve reflejado en los valores de completitud de inventario obtenidos para las tres ecorregiones, pudiéndose considerar como "completos" de acuerdo con Cardoso (2009). Ello nos lleva a pensar que los inventarios aquí obtenidos son buenos si se tiene en cuenta el balance costo/ tiempo con una única técnica de colecta. Así, los inventarios próximos al $70 \%$ de completitud son aceptables para grupos megadiversos, ya que elevar el esfuerzo de muestreo para superar apenas este valor es muy costoso en tiempo y dinero (Azevedo et al., 2014). Esto es apoyado por algunos autores (Azevedo et al., 2014; Porto et al., 2016) quienes han demostrado que es posible utilizar pocos métodos de muestreo y de bajo costo para alcanzar inventarios "completos", los cuales son útiles en planes de conservación y manejo (Pedley et al., 2016).

Coincidiendo con varios autores (Lubin, 1978; Avalos, Rubio, Bar, \& González, 2007; Hsieh \& Linsenmair, 2012; Azevedo et al., 2014; Bourass, Shaibi, Elkrwe, Ghana, \& Swehli, 2014), nuestros resultados demuestran que la estacionalidad contribuye a la composición de los ensambles de arañas. Las estaciones frías (otoño e invierno) evidencian ensambles de arañas diferentes respecto a las estaciones cálidas en las tres ecorregiones estudiadas. Por lo que los meses fríos albergan una cantidad significativa de especies en el área de estudio, lo cual contribuye a estimar la verdadera diversidad (Hiesh \& Linsenmair, 2012). Los muestreos efectuados en el otoño y el verano tienden a presentar un mayor número de especies en las tres ecorregiones, estos hallazgos concuerdan con los obtenidos por Chatzaki, Tricas, Markakis y Mylonas (1998) y Bourass et al. (2014) para otros ambientes áridos. En la Puna, la primavera también muestra poseer un alto número de especies, al igual que el otoño y el verano, resultados similares fueron obtenidos por Taucare-Ríos (2012) para ambientes de altura en Chile, a excepción del verano. De esa manera, las estaciones frías son claves en la zona en estudio a la hora de evaluar la diversidad de arañas y deberían ser tenidas en cuenta en futuras evaluaciones rápidas de diversidad y/o monitoreo, ya que, al igual que las estaciones cálidas (primavera y verano), también contribuyen con la diversidad de toda la región.

Los resultados obtenidos muestran que cada ecorregión estudiada presenta ensambles de arañas epigeas particulares, donde los sitios de Puna muestreados a mayor altitud (3 700-4 $000 \mathrm{msnm})$ registran los menores valores de riqueza de especies y abundancia de arañas. Estudios en Norteamérica (Bowden \& Buddle, 2010; Sudhikumar, 2015), Centroamérica (Hajian-Forooshani, Gonthier, Marín, Iverson, \& Perfecto, 2014) y Sudamérica (Janzen et al., 1976; Silva, 1992) han reportado el mismo patrón de disminución de la abundancia, la riqueza y/o la diversidad de arañas con el incremento de la altitud. Diversos factores han sido propuestos para explicar este patrón, tales como el bajo nivel de productividad (Lawton, MacGarvin, \& Heads, 1987; Jaksic, Marquet, \& González, 1997); la marcada variación diaria de la temperatura, la distribución irregular de las precipitaciones y la intensidad del viento (Polis \& Yamashita, 1991; Jaksic et al., 1997; Bizuet-Flores, Jiménez-Jiménez, Zavala-Hurtado, \& Corcuera, 2015).

Así mismo, este trabajo pone en evidencia que los cambios en la diversidad alfa y beta evaluados a nivel de especies también son reflejados al nivel taxonómico de familia, lo que podría ser de gran utilidad en futuros estudios de evaluaciones rápidas de biodiversidad. Además, la dominancia de las especies más abundantes de cada ecorregión es también evidenciada satisfactoriamente a nivel de familia. Zodariidae que está representada por L. birabeni resulta dominante en el Chaco Serrano, donde esta especie ha sido reportada como una indicadora de ambientes nativos (Torres, González-Reyes, Rodríguez-Artigas, \& Corronca, 2016). Por su lado, Lycosidae, Philodromidae, Anyphaenidae y Oonopidae quienes son dominantes en el Monte y la Puna, fueron reportadas como abundantes y diversas en las zonas secas de Argentina (Grismado et al., 2014). En la Puna chilena, Anyphaenidae y Philodromidae resultaron abundantes a los 4 000 msnm (Taucare-Ríos, 2012), este resultado 
coincide con los nuestros para la misma ecorregión del lado argentino. Diversos autores (Pik et al., 1999; Groc et al., 2010; Kallimanis et al., 2012; Timms et al., 2013) destacan la eficacia de la suficiencia taxonómica a la hora de realizar monitoreos y evaluaciones rápidas de biodiversidad, ya que su mayor beneficio es la reducción del costo monetario y el tiempo requerido (Williams \& Gaston, 1994; Groc et al., 2010; Souza et al., 2016), ambos factores críticos en los estudios de monitoreo (Kallimanis et al., 2012). De esta manera, la identificación a nivel de familias puede ser el mejor beneficio cuando el grupo en estudio contiene numerosas especies que son difíciles de identificar (Brennan et al., 2006), más aún, cuando el grupo es megadiverso y existe un número reducido de taxónomos especialistas en las distintas familias de arañas en el país y la región.

Los cambios en las características del ambiente y la utilización del hábitat por parte de las arañas (Foelix, 1996) queda reflejado en los cambios en la composición de los gremios. Por lo que en el contexto del monitoreo biológico, el enfoque de grupos funcionales también puede ser útil para reflejar cambios ecológicos inmediatos del ecosistema. El Chaco Serrano ofrece una mayor cantidad de recursos para las arañas especialistas, las cuales quedan pobremente representadas en el Monte y la Puna. En cambio, las arañas que se agrupan en otras cazadoras resultaron dominantes en la Puna y disminuyen su abundancia hacia el Monte y el Chaco. Este gremio junto con otros arácnidos, han sido reportados como los depredadores más importantes en las zonas áridas (Polis \& Yamashita, 1991; Taucare-Ríos, 2012).

Debido a la acelerada modificación y pérdida de los ambientes nativos en las ecorregiones de la provincia de Salta, este estudio brinda información básica que puede ser utilizada para futuras evaluaciones rápidas de biodiversidad contando con un grupo megadiverso como lo son las arañas. En primer lugar, el método de muestreo empleado (trampas de caída) permite una buena estimación de la diversidad alfa y beta, con diferencias significativas, de las comunidades de arañas epigeas. Se demuestra que la estacionalidad influye sobre la composición de la fauna de arañas epigeas, por lo que los factores que actúan a escala temporal deberían ser evaluados en los futuros estudios de biodiversidad y conservación en la región. Por otro lado, se destaca el nivel taxonómico de familias como potencial sustituto de las especies, asegurando su inclusión en futuros planes de conservación de la región y/o en monitoreo de la salud de los ambientes de estas ecorregiones.

\section{AGRADECIMIENTOS}

Este trabajo fue financiado por el Consejo de Investigación de la Universidad Nacional de Salta (CIUNSa) y el Instituto para el Estudio de la Biodiversidad de Invertebrados (IEBI-UNSa). Por último, agradecemos al Consejo Nacional de Investigaciones Científicas y Técnicas (CONICET) por su permanente colaboración.

\section{RESUMEN}

El incremento de la degradación de los hábitats naturales ha fortalecido la necesidad de conocer y evaluar los patrones de biodiversidad; siendo los inventarios biológicos y la suficiencia taxonómica herramientas que permiten describir y monitorear la diversidad en cortos períodos de tiempo. El estudio de la araneofauna en el norte Argentino es escaso en particular en ecorregiones donde se considera prioritario conservar sus ambientes. Las arañas son utilizadas como taxón indicador para comparar patrones de biodiversidad, donde el estudio de las familias y de los gremios puede potencialmente servir como sustitutos ecológicos de las especies dando indicación del uso del hábitat y de las estrategias de forrajeo. En este estudio, analizamos la diversidad alfa y beta de las comunidades de arañas epigeas en diferentes ecorregiones de la provincia de Salta (Chaco Serrano, Monte de Sierras y Bolsones, y Puna) utilizando trampas de caída y muestreos en las cuatro estaciones del año; y testeamos si el nivel taxonómico familia, en el caso de las arañas, puede actuar como potencial sustituto del nivel especie en las evaluaciones rápidas de biodiversidad. Para ello, en cada ecorregión seleccionamos tres sitios ampliamente separados que fueron muestreados durante el otoño, invierno, primavera y verano del 2005-2007. En cada sitio se colocaron 10 trampas de caída separadas por $10 \mathrm{~m}$ a lo largo de una transecta lineal y con una actividad de 7 días por estación. Se recolectaron 886 arañas de 100 especies/morfoespecies correspondientes a 19 familias. 
La ecorregión Chaco $(\mathrm{S}=56, \mathrm{~N}=495)$ reportó la mayor riqueza de especies y abundancia comparada con el Monte $(\mathrm{S}=44, \mathrm{~N}=262)$ y la Puna $(\mathrm{S}=23, \mathrm{~N}=129)$. La completitud del inventario obtenido para cada ecorregión fue completa, superando el $70 \%$. La diversidad alfa y beta evidenciaron que las comunidades ecorregionales de arañas fueron diferentes. Las estaciones más frías (otoño e invierno) demostraron ser claves a la hora de evaluar la diversidad de arañas en las ecorregiones, aportando a la diversidad regional conjuntamente con la diversidad de las estaciones cálidas (primavera y verano). Además, el nivel taxonómico de familia evidenció reflejar, de igual manera que el nivel especie, los cambios en la diversidad alfa y beta. El uso de trampas de caída en todas las estaciones del año y el reconocimiento de las familias de arañas para la fauna epigea son de gran utilidad para realizar futuras evaluaciones rápidas de biodiversidad en el área en estudio; permitiendo así incorporar este grupo taxonómico en los estudios de monitoreo de biodiversidad que se lleven a cabo en estas ecorregiones.

Palabras clave: diversidad de arañas, trampas de caída, ecorregión, estacionalidad, suficiencia taxonómica.

\section{REFERENCIAS}

Avalos, G., Rubio, G. D., Bar, M. E., \& González, A. (2007). Arañas (Arachnida: Araneae) asociadas a dos bosques degradados del Chaco húmedo en Corrientes, Argentina. Revista de Biología Tropical, 55, 899-909.

Azevedo, G. H., Faleiro, B. T., Magalhães, I. L., Benedetti, A. R., Oliveira, U., Pena Barbosa, J. P., Santos, M. T., Vilela, P. F., De Maria, M., \& Santos, A. J. (2014). Effectiveness of sampling methods and further sampling for accessing spider diversity: a case study in a Brazilian Atlantic rainforest fragment. Insect Conservation and Diversity, 7(4), 381-391.

Bertonatti, C., \& Corcuera, J. (2000). Situación Ambiental Argentina. 2000. Buenos Aires: Fundación Vida Silvestre.

Bizuet-Flores, M. Y., Jiménez-Jiménez, M. L., Zavala-Hurtado, A., \& Corcuera, P. (2015). Diversity patterns of ground dwelling spiders (Arachnida: Araneae) in five prevailing plant communities of the Cuatro Ciénegas Basin, Coahuila, Mexico. Revista Mexicana de Biodiversidad, 86, 153-163.

Bourass, E. M., Shaibi, T., Elkrwe, H. M., Ghana, S., \& Swehli, A. I. (2014). Spider fauna (Araneae) of Abu Ghilan National Nark, north-western Libya. Indian Society of Arachnology, 3(2), 6-16.

Bowden, J. J., \& Buddle, C. M. (2010). Spider assemblages across elevational and latitudinal gradients in the Yukon Territory, Canada. Arctic, 63(3), 261-272.
Brennan, K. E., Ashby, L., Majer, J. D., Moir, M. L., \& Koch, J. M. (2006). Simplifying assessment of forest management practices for invertebrates: How effective are higher taxon and habitat surrogates for spiders following prescribed burning? Forest Ecology and Management, 231(1), 138-154.

Buchholz, S. (2010). Ground spider assemblages as indicators for habitat structure in inland sand ecosystems. Biodiversity and Conservation, 19, 2565-2595.

Cabrera, A. L. (1957). La vegetación de la Puna Argentina. Revista de Investigaciones Agricolas, 11(4), 317-512.

Cardoso, P. (2009). Standardization and optimization of arthropod inventories-the case of Iberian spiders. Biodiversity and Conservation, 18, 3949-3962.

Cardoso, P., Pekár, S., Jocque, R., \& Coddington, J. A. (2011). Global patterns of guild composition and functional diversity of spiders. PLOS ONE, 6, e21710. DOI:10.1371/journal.pone.0021710

Cardoso, P., Scharff, N., Gaspar, C., Henriques, S. S., Carvalho, R., Castro, P. H., Schmidt JB, Silva I, Szuts T, De Castro A., \& Crespo, L. C. (2008). Rapid biodiversity assessment of spiders (Araneae) using semi-quantitative sampling: a case study in a Mediterranean forest. Insect Conservation and Diversity, 1(2), 71-84.

Cardoso, P., Silva, I., De Oliveira, N. G., \& Serrano, A. R. (2007). Seasonality of spiders (Araneae) in Mediterranean ecosystems and its implications in the optimum sampling period. Ecological Entomology, 32(5), 516-526.

Castanheira, P., Pérez-González, A., \& Baptista, R. L. (2016). Spider diversity (Arachnida: Araneae) in Atlantic Forest areas at Pedra Branca State Park, Rio de Janeiro, Brazil. Biodiversity Data Journal, 4, e7055. DOI: 10.3897/BDJ.4.e7055

Cava, M. B., Corronca, J. A., \& Echeverría, A. J. (2013). Alpha and beta arthropods diversity from the different environments of Parque Nacional Los Cardones, Salta, Argentina. Revista de Biología Tropical, 61(4), 1785-1798.

Chatzaki, M., Trichas, A., Markakis, G., \& Mylonas, M. (1998). Seasonal activity of the ground spider fauna in a Mediterranean ecosystem (Mt Youchtas, Crete, Grece). Proceedings of the 17th European Colloquium of Arachnology, 235-243.

Chao, A., \& Jost, L. (2012). Coverage-based rarefaction and extrapolation: standardizing samples by completeness rather than size. Ecology, 93(12), 2533-2547.

Chao, A., \& Shen, T. (2003-2005). Program SPADE. Species Prediction and Diversity Estimation. http://chao. stat.nthu.edu.tw 
Chao, A., Ma, K. H., \& Hsieh, T. C. (2016). iNEXT (iNterpolation and EXTrapolation). Software for Interpolation and Extrapolation of Species Diversity. http:// chao.stat.nthu.edu.tw/wordpress/software_download/

Chébez, J. C. (2005). Guía de las reservas naturales de la Argentina. Noroeste. Buenos Aires: Albatros.

Colwell, R. K. (2013). EstimateS: Statistical estimation of species richness and shared species from samples (Version 9.1). http://viceroy.eeb.uconn.edu/estimates/

Ellis, D. (1985). Taxonomic sufficiency in pollution assessment. Marine Pollution Bulletin, 16(12), 459.

Escorcia, R. Y., Martínez, N. J., \& Silva, J. P. (2012). Study of spiders' diversity in a tropical dry forest (bs-t) in sabanalarga, Atlántico, Colombia. Boletín Cientifico. Centro de Museos. Museo de Historia Natural, 16(1), 247-260.

Foelix, R. F. (1996). Biology of Spiders (2nd Ed.). New York: Oxford University Press.

González Reyes, A. X., Corronca, J. A., \& Arroyo, N. C. (2012). Differences in alpha and beta diversities of epigeous arthropod assemblages in two ecoregions of northwestern Argentina. Zoological Studies, 51(8), 1367-1379.

Grau, R. H., Gasparri, N. I., \& Aide, M. (2008). Balancing food production and nature conservation in the Neotropical dry forests of northern Argentina. Global Change Biology, 14(5), 985-997.

Grismado, C. J., Ramírez, M. J., \& Izquierdo, M. A. (2014). Araneae: Taxonomía, diversidad y clave de identificación de familias de la Argentina. En S. Roig-Juñent, L. E. Claps, \& J. J. Morrone (Eds.), Biodiversidad de Artrópodos Argentinos Vol. 3 (pp. 55-93). San Miguel de Tucumán: Editorial INSUEUniversidad Nacional de Tucumán.

Groc, S., Delabie, J. H., Longino, J. T., Orivel, J., Majer, J. D., Vasconcelos, H. L., \& Dejean, A. (2010). A new method based on taxonomic sufficiency to simplify studies on Neotropical ant assemblages. Biological Conservation, 143(11), 2832-2839.

Haddad, C. R., Honiball, A. S., Dippenaar-Schoeman, A. S., Slotow, R., \& Van Rensburg, B. J. (2009). Spiders as potential indicators of elephant-induced habitat changes in endemic sand forest, Maputaland, South Africa. African Journal of Ecology, 48(2), 446-460.

Hajian-Forooshani, Z., Gonthier, D. J., Marín, L., Iverson, A. L., \& Perfecto, I. (2014). Changes in species diversity of arboreal spiders in Mexican coffee agroecosystems: untangling the web of local and landscape influences driving diversity. PeerJ, 2, e623. https://doi.org/10.7717/peerj.623

Hammer, Ø., Harper, D.A.T., \& Ryan, P. D. (2001). PAST: Paleontological statistics software package for education and data analysis. Paleontologia Electronica, 4(1), 9 .

Hsieh, Y. L., \& Linsenmair, K. E. (2011). Underestimated spider diversity in a temperate beech forest. Biodiversity and Conservation, 20(13), 2953-2965.

Hsieh, Y. L., \& Linsenmair, K. E. (2012). Seasonal dynamics of arboreal spider diversity in a temperate forest. Ecology and Evolution, 2(4), 768-777.

Hsieh, Y. L., Lin, Y.-S., \& Tso, I.-M. (2003). Ground spider diversity in the Kenting uplifted coral reef forest, Taiwan: a comparison between habitats receiving various disturbances. Biodiversity and Conservation, $12,2173-2194$

Jaksic, F., Marquet, P., \& González, H. (1997). Una perspectiva ecológica sobre el uso del agua en el Norte Grande. Estudios Públicos, 68, 171-195.

Janzen, D. H., Ataroff, M., Fariñas, M., Reyes, S., Rincon, N., Soler, A., Soriano, P., \& Vera, M. (1976). Changes in the arthropod community along an elevational transect in the Venezuelan Andes. Biotropica, 8(3), 193-203.

Jiménez-Valverde, A., \& Lobo, J. M. (2007). Determinants of local spider (Araneidae and Thomisidae) species richness on a regional scale: climate and altitude vs. habitat structure. Ecological Entomology, 32(1), 113-122.

Kallimanis, A. S., Mazaris, A. D., Tsakanikas, D., Dimopoulos, P., Pantis, J. D., \& Sgardelis, S. P. (2012). Efficient biodiversity monitoring: Which taxonomic level to study? Ecological Indicators, 15(1), 100-104.

Krell, F. T. (2004). Parataxonomy vs. taxonomy in biodiversity studies-pitfalls and applicability of 'morphospecies' sorting. Biodiversity and Conservation, 13(4), 795-812.

Labraga, J. C., \& Villalba, R. (2009). Climate in the Monte Desert: past trends, present conditions, and future projections. Journal of Arid Environments, 73(2), 154-163.

Landeiro, V. L., Bini, L. M., Costa, F. R., Franklin, E., Nogueira, A., de Souza, J. L., Moraes, J., \& Magnusson, W. E. (2012). How far can we go in simplifying biomonitoring assessments? An integrated analysis of taxonomic surrogacy, taxonomic sufficiency and numerical resolution in a megadiverse region. Ecological Indicators, 23, 366-373.

Lawton, J. H., MacGarvin, M., \& Heads, P. A. (1987). Effects of altitude on the abundance and species richness of insect herbivores on bracken. Journal of Animal Ecology, 56, 147-160.

Lubin, Y. D. (1978). Seasonal abundance and diversity of web-building spiders in relation to habitat structure 
on Barro Colorado Island, Panama. Journal of Arachnology, 6, 31-51.

Magnusson, W. E. (2004). Ecoregion as a pragmatic tool. Conservation Biology, 18, 4-5.

McCune, B., \& Mefford, M. J. (1999). PC-Ord. Multivariate Analysis of Ecological Data (Version 5.0). Gleneden Beach, Oregon, USA: MjM Software Design.

Moglia, J. G., \& Giménez, A. (1998). Rasgos característicos del hidrosistema de las leñosas de la Región Chaqueña. Revista de Investigaciones Agrarias - Sistemas y Recursos Forestales. España, 7, 41-53.

Morello, J. (2012). Ecorregión del Chaco Seco. En J. Morello, S. D. Matteucci, A. F. Rodriguez, \& M. E. Silva (Eds.), Ecorregiones y Complejos Ecosistémicos Argentinos (pp. 151-204). Buenos Aires: Orientación Gráfica.

Moreno, C. E., \& Halffter, G. (2000). Assessing the completeness of bat biodiversity inventories using species accumulation curves. Journal of Applied Ecology, 37(1), 149-158.

Mosca Torres, M. E., \& Puig, S. (2010). Seasonal diet of vicuñas in the Los Andes protected area (Salta, Argentina): Are they optimal foragers? Journal of Arid Environments, 74(4), 450-457.

Muñoz-Gutiérrez, J. A., Roussea, G. X., Andrade-Silva, J., \& Delabie, J. H. C. (2017) Ants' higher taxa as surrogates of species richness in a chronosequence of fallows, old-grown forests and agroforestry systems in the Eastern Amazon, Brazil. Revista de Biologia Tropical, 65(1), 279-291.

New, T. R. (1996). Taxonomic focus and quality control in insect surveys for biodiversity conservation. Australian Journal of Entomology, 35(2), 97-106.

Olson, D. M., \& Dinerstein, E. (1998). The global 200: a representation approach to conserving Earth's most biologically valuable ecoregions. Conservation Biology, 12, 502-515.

Olson, D. M., \& Dinerstein, E. (2002). The Global 200: priority ecoregions for global conservation. Annals of the Missouri Botanical garden, 89, 199-224.

Pedley, S. M., Oxbrough, A., Martin, R. D., Irwin, S., Kelly, T. C., \& O'Halloran, J. (2016). Can groundbased assessments of forest biodiversity reflect the biological condition of canopy assemblages? Forest Ecology and Management, 359, 190-198.

Pik, A. J., Oliver, I. A. N., \& Beattie, A. J. (1999). Taxonomic sufficiency in ecological studies of terrestrial invertebrates. Australian Journal of Ecology, $24(5), 555-562$.
Piquer-Rodríguez, M., Torella, S., Gavier-Pizarro, G., Volante, J., Somma, D., Ginzburg, R., \& Kuemmerle, T. (2015). Effects of past and future land conversions on forest connectivity in the Argentine Chaco. Landscape Ecology, 30(5), 817-833.

Polis, G. A., \& Yamashita, T. (1991). The ecology and importance of predaceous arthropods in desert communities. In G. A. Polis (Ed.), The ecology of desert communities (pp. 180-222). Tucson: The University of Arizona Press.

Porto, W., Pequeno, P. A. L., \& Tourinho, A. L. (2016). When less means more: Reduction of both effort and survey methods boosts efficiency and diversity of harvestmen in a tropical forest. Ecological Indicators, 69, 771-779.

Reboratti, C. (2006). Situación ambiental en las ecorregiones Puna y Altos Andes. En A. Brown, U. Martínez Ortiz, M. Acerbi, \& J. Corcuera (Eds.), La Situación Ambiental Argentina 2005 (pp. 28-31). Buenos Aires: Fundación Vida Silvestre Argentina.

Rodriguez-Artigas, S. M., Ballester, R., \& Corronca, J. A. (2016). Factors that influence the beta-diversity of spider communities in northwestern Argentinean Grasslands. PeerJ, 4, e1946. DOI:10.7717/peerj.1946

Rubio, G. D., \& Moreno, C. E. (2010). Orb-weaving spider diversity in the Iberá Marshlands, Argentina. Neotropical Entomology, 39(4), 496-505.

Rundel, P., Villagra, P. E., Dillon, M. O., Roig-Juñent, S. A., \& Debandi, G. (2007). Arid and SemiArid Ecosystems. In T. T. Veblen, K. Young, \& A. Orme (Eds.), The physical geography of South America (pp. 158-183). New York: Oxford University Press.

Siewers, J., Schirmel, J., \& Buchholz, S. (2014). The efficiency of pitfall traps as a method of sampling epigeal arthropods in litter rich forest habitats. European Journal of Entomology, 111(1), 69-74.

Silva, D. (1992). Observations on the diversity and distribution of the spiders of Peruvian montane forests. Memorias del Museo de Historia Natural, Universidad Nacional Mayor de San Marcos, 21, 31-37.

Souza, J. L. P., Baccaro, F. B., Landeiro, V. L., Franklin, E., Magnusson, W. E., Pequeno, P. A. C. L., \& Fernandes, I. O. (2016). Taxonomic sufficiency and indicator taxa reduce sampling costs and increase monitoring effectiveness for ants. Diversity and Distributions, 22(1), 111-122.

Spence, J. R., \& Niemelä, J. (1994). Sampling carabid assemblages with pitfall traps: the madness and the method. Canadian Entomologist, 126, 881-894. 
Sudhikumar, A. V. (2015). Distribution pattern of spiders along an elevational gradient in Nelliyampathy Hill Ranges of the Western Ghats, Kerala, India. International Journal of Science and Research, 4(7), 170-173.

Taucare-Ríos, A. (2012). Arañas epigeas (Araneae) en el Parque Nacional Volcán Isluga, Altiplano chileno. Brenesia, 78, 50-57.

Timms, L. L., Bowden, J. J., Summerville, K. S., \& Buddle, C. M. (2013). Does species-level resolution matter? Taxonomic sufficiency in terrestrial arthropod biodiversity studies. Insect Conservation and Diversity, 6(4), 453-462.

Torres, V. M., González-Reyes, A. X., Rodriguez-Artigas, S. M., \& Corronca, J. A. (2016). Efectos del disturbio antrópico sobre las poblaciones de Leprolochus birabeni (Araneae, Zodariidae) en el Chaco Seco del noroeste de Argentina. Iheringia, Série Zoologia, 106, e2016009. DOI:10.1590/1678-4766e2016009

Whitmore, C., Crouch, T. E., \& Slotow, R. H. (2002). Conservation of biodiversity in urban environments: invertebrates on structurally enhanced road islands. African Entomology, 10, 113-126.

Williams, P. H., \& Gaston, K. J. (1994). Measuring more of biodiversity: can higher-taxon richness predict wholesale species richness? Biological Conservation, 67(3), 211-217.

World Spider Catalog. (2017). World Spider Catalog, Version 17.5. Natural History Museum Bern. Obtenido de http://wsc.nmbe.ch

See Digital Appendix at: / Ver Apéndice digital en:

revistas.ucr.ac.cr 